\title{
GST Networks: Learning Emergent Spatiotemporal Correlations ${ }^{1}$
}

\author{
Chaitanya Tumuluri Chilukuri K. Mohan Alok N. Choudhary \\ tumuluri@cat.syr.edu \\ ckmohan@syr.edu \\ choudhar@cat.syr.edu \\ 121 Link Hall \\ 2-120 CST \\ 121 Link Hall
}

Syracuse University, Syracuse, NY 13244

\begin{abstract}
This paper presents two novel (GIST and GEST) networks, which combine unsupervised featureextraction and Hebbian learning, for tracking emergent correlations in the evolution of spatiotemporal distributions. The networks were successfully tested on the challenging Data Mapping problem, using an execution driven simulation of their implementation in hardware.
\end{abstract}

\section{Introduction}

Several important and difficult problems require tracking emergent spatio-temporal correlations among useful features of time-varying data distributions, where it may not be possible to obtain $a$ priori analytical characterization of the processes generating the data. Data Mapping is one such problem where disjoint sets of data must be mapped to processors in a multiprocessor system to minimize off-processor data accesses. This requires (a) modeling emergent distribution of data references and (b) tracking correlations in the distribution of references. We extend Fritzke's unsupervised Growing Cell Structures (GCS) network [1] for these purposes to produce two new networks models: the GCS Instantaneous Spatio-Temporal (GIST) network and the GCS Epochal Spatio-Temporal (GEST) network, together referred to as the GST networks. We demonstrate their success in learning the spatiotemporal dynamics on the challenging Data Mapping problem.

\section{GST Spatial Mapping Dynamics}

The GST network is a 2-dimensional network of neurons forming planar maps, whose topology always consist of triangles. Figure 1 illustrates the network and highlights the various connectivities among neurons. The lateral weight $W_{i j}$, connecting neuron $j$ to neuron $i$, records emergent temporal correlations in their activations. A Signal Receptivity Meter (SRM) records the number of hits to each neuron over an epoch and is an enhancement over the Signal Frequency Counter (SFC) of the GCS network.

The GST spatial dynamics position neurons at cluster centers in the input Signal Vector Space (SVS) during both the training and the production modes of operation. The network uses training

\footnotetext{
${ }^{1}$ This research was supported in part by an NSF Young Investigator Award CCR-9357840.
} 
data to approximately map time-varying distributions in the offline training mode. The map is refined online using actual incoming data during the production mode. Network performance parameters, evaluated at epochal checkpoints, are used to decide if the network needs offline retraining. Both modes involve:

1. Input vector quantization and subsequent input weight ( $W^{I}$ in Figure 1) adaptations as in Kohonen's SOM [2] with the difference that the adaptation rates are fixed (i.e. nonstochastic).

2. The SRMs of the winner neuron and all its neighbors are adapted as opposed to the GCS dynamics which adapt the SFC of only the winner neuron.

The GST architectural dynamics define the structural adaptations in the network which only occur under conditions wherein the network-wide average quantization error changes less than $10 \%$ across successive epochs. Architectural adaptations under such conditions of positional equilibrium involve:

1. Addition of a neuron, as a topological neighbor of the neuron with the highest average quantization error, to increase map resolution and decrease network-wide average quantization error.

2. Deletion of one superflous neuron with the least relative SRM value, $\tau_{i} / \sum_{j}\left(\tau_{j}\right)$, (and any neighbors not part of at least one topological triangle [1]), to coarsen resolution in areas of low input probability.

\section{GST Temporal Mapping Dynamics}

The production mode temporal dynamics of the GIST and GEST networks involve: (a) different lateral weight update schemes but (b) the same computations of temporal correlations in neuronal activations. The computed temporal correlations are used to obtain the total probability of activation, $P_{i}$, of neuron $i$. $P_{i}$ is termed the Signal Utility of inputs mapped to this neuron and measures the usefulness of this data-cluster in the temporal evolution of the SVS distribution. The total probability, $P_{i}$ is given by:

$$
P_{i}=\sum_{j}\left(P_{(i \mid j)} \times \hat{p}_{j}\right)
$$

$P_{(i \mid j)}$ is the conditional probability of correlations in the evolution of cluster $i$ with respect to the evolution of cluster $j$ and is given by: $P_{(i \mid j)}=W_{i j} / \sum_{k} W_{k j}$. The a priori probability of inputs quantized by neuron $j$ is: $\hat{p_{j}}=\tau_{j} / \sum_{k} \tau_{k}$, where $\tau_{j}$ is the SRM of neuron $j$. 
The GIST lateral weight, $W_{i j}$, records the frequency with which first neuron $j$ and then neuron $i$ are activated (i.e. first-order correlations). These weights are used in computing the $P_{(i \mid j)}$ values which yield the various $P_{i}$.

The GEST lateral weights record higher-order correlations using an adaptation of the Drive Reinforcement Theory (DRT) learning equation [3]. The correlation interval, $\Lambda=\sum_{k} \tau_{k} /\left|\bar{\tau}-\sigma_{\tau}\right|$, represents the number of presentations after which weight updates are performed. $\bar{\tau}$ is the networkwide average SRM value and $\sigma_{\tau}$ is the standard deviation. The lateral weight update rule at the end of the interval $[t-\Lambda, t]$ is:

$$
\Delta W_{i j}(t)=\Delta P_{i}(t) \times \sum_{n}\left(\left|W_{i j}(t-n \Lambda)\right| \times \triangle \hat{p}_{j}(t-n \Lambda)\right)
$$

\section{GST Application: Problem Formulation}

The allocation of computations on data to processors, in data mapping, requires data-dependency information. The problem of modeling temporally emergent dependencies is solved by determining temporally emergent probabilities of correlations in processor references to data elements.

The test case involves mapping data in an ecological simulation, "WaTor," of sharks and minnows in a toroidal ocean [4]. WaTor exhibits rapidly varying localities among slowly varying dataset sizes. The simulation parameters include a $200 \times 75$ ocean grid with 5000 sharks and 1563 minnows. The simulation runs for 10 iterations using 4, 8, 16 and 24 processors. Each application processor's task partition consists of minnows distributed in the toroidal ocean mesh. Remote references would be minimized if each processor's partition consists of neighboring minnows as WaTor computations require near-neighbor interactions. Hence, execution locality is measured by the average Euclidean distance (in the ocean) seperating minnows within each processor's task partition.

\section{The Solution: Simulation Model and Application}

Memory is abstracted as consisting of indivisible and contiguous Atomic Memory Units (AMUs). Among descriptors associated with each AMU, the $A M U$ Utility records the probability of local references to the AMU. Ongoing local computation induces changes in AMU descriptors producing non-stationary SVS distributions at each processor. For example, the AMU Utility varies due to increases (decreases) in the frequency of references to various AMUs as they are inducted (expelled) from the local working-set.

A GST (either GIST or GEST) network is used at each processor to capture spatial maps of AMU distributions in the local SVS from the processor reference streams. The Signal Utility of the neuron quantizing an AMU is assigned its AMU Utility. Offline training sets for networks are 


\begin{tabular}{||l|l||}
\hline GST Activities & Execution Speed \\
\hline \hline Training Vector Generation $\left(T_{T V e c t}\right)$ & $7 \mathrm{KSR} 1$ cycles \\
\hline Snoop Vector Generation $\left(T_{S V e c t}\right)$ & $62 \mathrm{KSR} 1$ cycles \\
\hline Input Vector Quantization $\left(T_{Q u a n t}\right)$ & $10 \mathrm{KSR} 1$ cycles \\
\hline GIST Lateral Weights Update $\left(T_{I M}\right)$ & $34 \mathrm{KSR} 1$ cycles \\
\hline GEST Lateral Weights Update $\left(T_{E M}\right)$ & $\left(n^{2}+30 n\right) \mathrm{KSR} 1$ cycles \\
\hline Neuron Addition $\left(T_{A d d}\right)$ & $100 \mathrm{KSR} 1$ cycles \\
\hline Neuron Deletion $\left(T_{D e l}\right)$ & $200 \mathrm{KSR} 1$ cycles \\
\hline
\end{tabular}

Table 1: GST Simulation Timing Parameters. $n$ in the $T_{E M}$ timing refers to the network size.

derived from past processor references and the current contents of local memory. In this context, $\hat{p}_{j}$ provides an a priori frequency based measure of the probablity that AMU-cluster $j$ contains AMUs of the current local working set. Also, $P_{(i \mid j)}$ can be viewed as the conditional probability that references to AMUs in cluster $i$ are correlated with references to AMUs in cluster $j$. Thus, $P_{i}$ is the probability of the presence of references belonging to cluster $i$ in the reference stream.

The simulations assume an on-chip GST coprocessor unit per processor in the multiprocessor system. The execution driven simulation, implemented on the KSR1 [5], simulates concurrent execution of the GST coprocessor and the WaTor application. The essential timings for GST activities are given in Table 1 , and have been derived from simulations and results in $[6,5,7]$.

\section{Simulation Results}

The experimental results show that the GST networks successfully track the varying locality characteristics online and produce increasingly better execution locality.

- GST Sampling Efficiency: References from the co-executing application processor are discarded during training and so the sampling efficiency, [No. of Refs. Processed]/[Total No. of Refs.], evaluates the online tracking ability. The sampling efficiency ranges between $90 \%$ to $100 \%$, indicating short offline training phases as in Figure 2 for the GEST network. The GIST sampling efficiency (not shown due to space limitations) was higher in comparison with the GEST network (primarily because $T_{E M}>T_{I M}$ usually), but suffered more high-frequency variations.

- WaTor Application Performance: The execution timings in Table 2 show scalable WaTor performance and indicate that Minnow updates were responsible for $75 \%$ to $90 \%$ of the total execution time. The GIST locality measure plotted in Figure 3 shows increasingly better execution locality wherein the inter-minnow distances decrease from an initial high of 75 to level off at about 40 . Hence, processor partitions achieved increasing contiguity 


\begin{tabular}{||c|c|c|c|c||}
\hline & \multicolumn{2}{|c|}{ Minnow Loop } & \multicolumn{2}{c||}{ Shark Loop } \\
\hline Run Size & GIST Run & GEST Run & GIST Run & GEST Run \\
\hline \hline 4 Processors & $23.695952 \mathrm{sec}$ & $21.768551 \mathrm{sec}$ & $4.679540 \mathrm{sec}$ & $3.528160 \mathrm{sec}$ \\
\hline 8 Processors & $13.460341 \mathrm{sec}$ & $13.248189 \mathrm{sec}$ & $2.312860 \mathrm{sec}$ & $2.040482 \mathrm{sec}$ \\
\hline 16 Processors & $8.473986 \mathrm{sec}$ & $6.6604046 \mathrm{sec}$ & $1.409917 \mathrm{sec}$ & $1.273765 \mathrm{sec}$ \\
\hline 24 Processors & $6.034877 \mathrm{sec}$ & $4.6708921 \mathrm{sec}$ & $1.140522 \mathrm{sec}$ & $1.208808 \mathrm{sec}$ \\
\hline
\end{tabular}

Table 2: WaTor Execution Timings (using GIST and GEST networks)

and compactness in spite of the increasing dataset sizes. This is also evident in the GEST network runs plotted in Figure 4 which shows an almost monotonic decrease in the inter-task distances.

\section{Conclusions}

The simulation results (in Section 6) show that both the GIST and GEST networks converge to stable maps of emergent non-stationary distributions without undue loss in their sampling efficiencies. Higher-order correlations are useful for smoothing high-frequency variations, and extract long-term low-frequency correlations in neuronal activations. Hence, the GEST network is better at tracking the long-range evolution of non-stationary distributions as seen in the almost monotonic improvements in locality in the GEST network runs (Figure 4). However, GEST network sampling efficiency was lower in comparison with that of the the GIST network.

\section{References}

[1] B. M. Fritzke, "Growing Cell Structures - A Self-organizing Network for Unsupervised and Supervised Learning," Neural Networks, vol. 7, no. 9, pp. 1441-1460, 1993.

[2] T. Kohonen, Self-Organization and Associative Memory. Heidelberg, Germany: SpringerVerlag, third ed., 1989.

[3] M. Caudill, "Neural Networks Primer, Part VII," AI Expert, pp. 55-58, May 1989.

[4] A. K. Dewdney, "Computer Recreations," Scientific American, pp. 14-22, December 1984.

[5] Kendall Square Research, KSR/Series Principles of Operation, March 1994.

[6] R. H. Saavedra, R. S. Gaines, and M. J. Carlton, "Micro Benchmark Analysis of the KSR1," in Proc. of SUPERCOMPUTING''93, pp. 202-213, September 1993.

[7] H. Speckmann, P. Thole, and W. Rosenstiel, "A COprocessor for KOhonen's Selforganizing Map (COKOS)," in Procs. 1993 International Joint Conference on Neural Networks, pp. 1951-1954, 1993. 

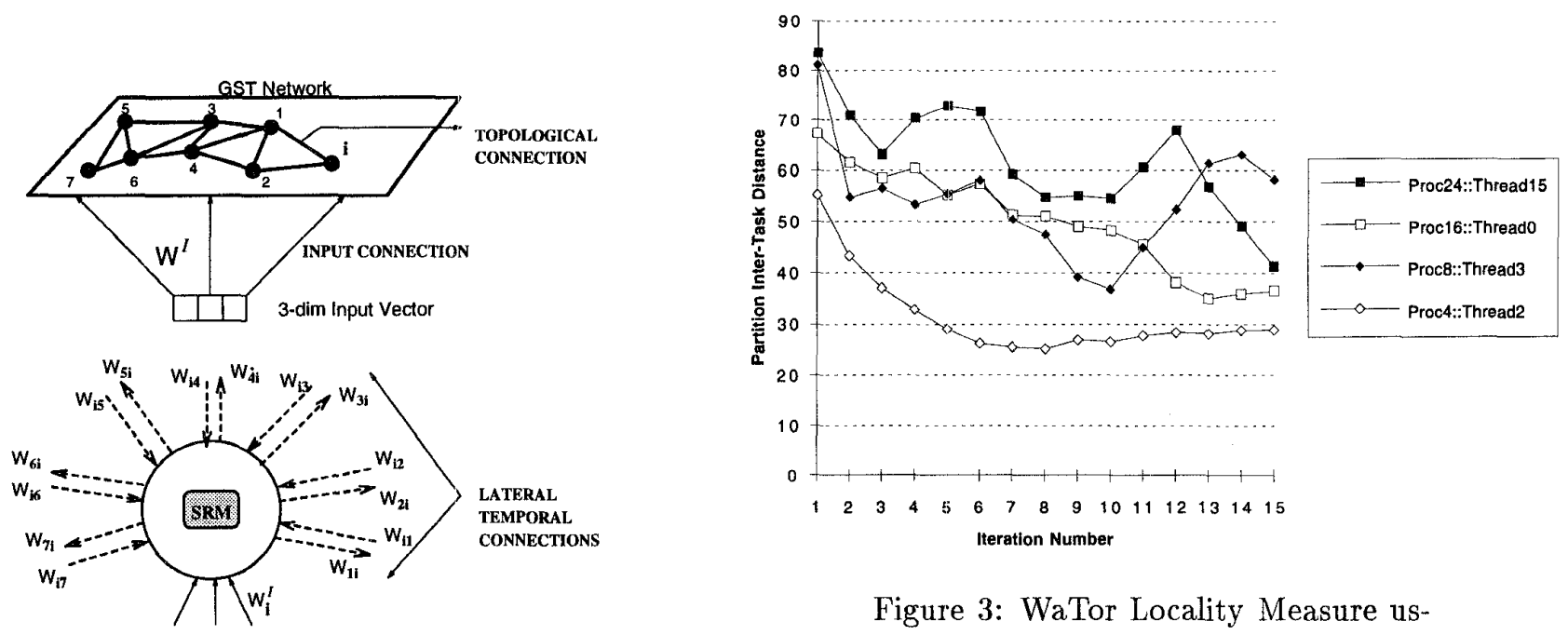

Figure 3: WaTor Locality Measure using GIST networks(15 iteration run) :: Thread Intra-Task Distances

Proc $x:$ Thread $y \Rightarrow$ Locality Characteristics of Thread\#y in an $x$ processor run
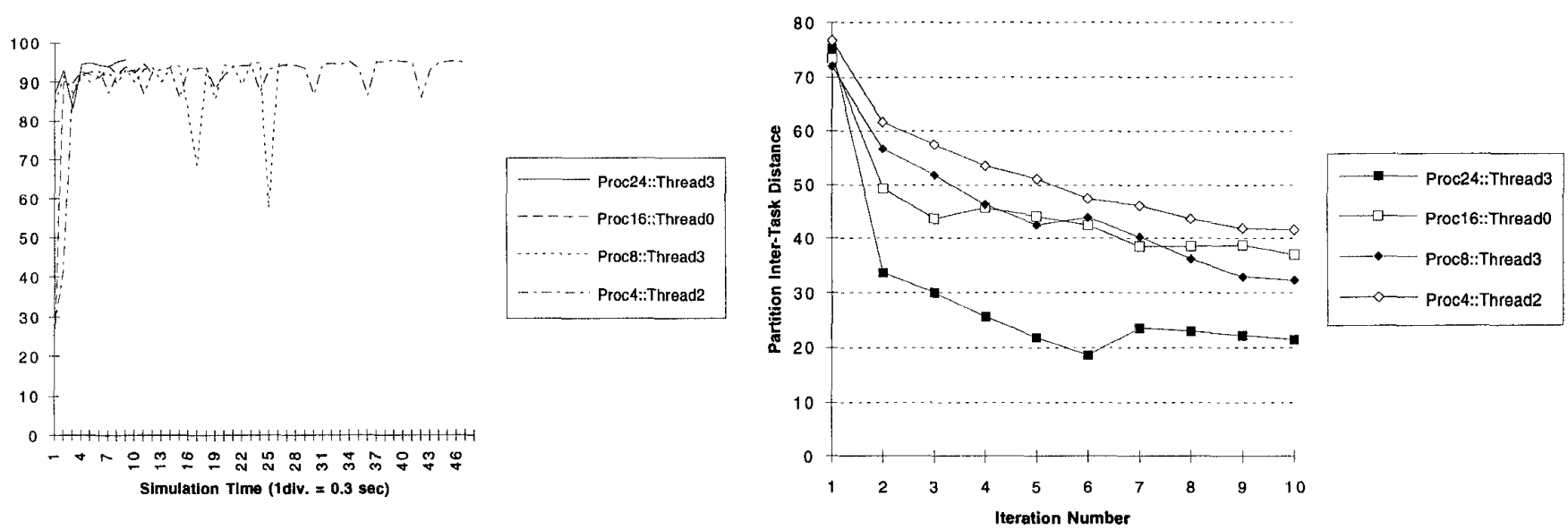

Figure 2: WaTor (10 iteration run) :: GEST Network Sampling Efficiency Proc $x:$ Thread $y \Rightarrow$ GEST network for Application Thread\#y in an $x$ processor run

Figure 4: WaTor Locality Measure using GEST networks(10 iteration run) :: Thread Intra-Task Distances Proc $x::$ Thread $y \Rightarrow$ Locality Characteristics of Thread\#y in an $x$ processor run 